\title{
Comparative Study of Wavelet Image Compression: JPEG2000 Standart
}

\author{
Ansam Ennaciri ${ }^{* 1}$, Mohammed Erritali ${ }^{2}$, Mustapha Mabrouki ${ }^{3}$, Jamaa Bengourram ${ }^{4}$ \\ Faculty of Sciences and Techniques, Sultan MoulaySlimane University \\ B.P: 523, BENI MELLAL, MOROCCO \\ ${ }^{1,3,4}$ Departement of Industrial Engineering, Faculty of Sciences and Techniques, \\ Sultan Moulay Slimane University \\ ${ }^{2}$ TIAD laboratory, Departement of Computer Sciences, Faculty of Sciences and Techniques, \\ Sultan Moulay Slimane University \\ ${ }^{*}$ Corresponding author, e-mail: ennaciri.ansam@gmail.com ${ }^{1}$, m.erritali@usms.ma², \\ mus_mabrouki@yahoo.fr ${ }^{3}$, bengoram@yahoo.fr ${ }^{4}$
}

\begin{abstract}
The objective of this paper is to study the main characteristics of wavelets that affect the image compression by using the discrete wavelet transform and lead to an image data compression while preserving the essential quality of the original image. This implies a good compromise between the image compression ratio and the PSNR (Peak Signal Noise Ration).
\end{abstract}

Keywords: compression, wavelet transform, haar, PSNR (peak signal noise ration)

Copyright () 2015 Institute of Advanced Engineering and Science. All rights reserved.

\section{Introduction}

Nowadays, data compression has a significant importance especially in the IT (Information technology) domain and internet data exchange. This importance is related to the limited bandwidth that an internet server offers. For instance, a data volume of twenty megabytes cannot be sent through a mail server due to its bandwidth which is smaller than the data volume. Hence, the need for compression is essential in order to reduce the costs and the ressources.

In this project we are interested in the compression of images with losses based specifically on compression wavelet transform [1] better known by the name of the JPEG2000 standard (Joint Photographic Experts Group 2000) [2, 3]. During this work it was entrusted us to treat different stages of image compression using discrete wavelet transform (DWT: discrete wavelet transform) and to make a comparison between its algorithms.

The image compression reduces the number of bits needed in order to represent an image [4], therefore the amount of memory required to store the data will be reduced. It also reduces the amount of time required to transmit the data via a communication link at a given rate.

The purpose of compression is to reduce the amount of memory needed for storing an image, therefore reduce the time of its transmission. This compression can either keep the image intact, known as the lossless compression mode, or allow an image degradation and therefore reduce the memory footprint, this is known as the lossy compression mode. The first mode is limited to the third order compression factors (the ratiobetween the original size and the compressed size), while on the other hand, the second mode of compression allows a much greater order in the cost of the image degradation.

Image compression can be done in two ways either losslessor lossy. The first compression method may be used to guard a high accuracy, such as for medical images like entropy coding [5], the repetition coding method and adaptive dictionary such as LZW algorithms [6].For the second method that of lossy, is used for artificial imagesby the discrete cosine transform [6], or the fractal compression [7]. Indeed when particular uses lossy compression methods at low bit rates, it presents compression defects. This is because it is used in normal images such as photos in applications where a minor loss of loyalty (sometimes imperceptible) is acceptable to reach a meaningful minimization in the binary debit. 
An anothoer known methods for lossly image compression can be done,by using the discrete wavelet transformation. Transform coding comprises applying the transformation to the image, followed by a quantization and entropy coding.

In fact, the wavelet transform (used in DWT) has the advantage of bringing out both the wide variations and details of the analyzed object, that does not allow the Fourier transform (used in DCT).

When approaching the topic of image compression, the first standard that comes to mind is JPEG (Joint Photographic Experts Group). The compression algorithm JPEG2000 standard (Joint Photographic Experts Group 2000) [4, 8] provides more benefits than JPEG because of the resemblance in processing steps with one major difference: instead of using the DCT(Discrete Cosine Transform), JPEG2000 standard (Joint Photographic Experts Group 2000) [2, 3] uses the DWT(Discrete Cosine Transform which offers us more benefits.

Our contributions include having a good compromise between compression rate and the signal to noise ratio for proper use of the image compression.

This paper is organized as follows: Section 2 explain the fundamentals of wavelet transform. Section 3 describes the basic principles of Haar algorithm. Section 4demonstrates the experimental results and discussions. Finally Section 5 concludes the paper.

\section{Wavelet Transform}

The Wavelet Transform is similar to the Fourier Transform (and more to the Local Fourier Transform) with a merit function completely different [1]. In brief, the Wavelet Transform can be expressed with the following equation [1]:

$$
F(a, b)=\int_{-\infty}^{\infty} f(x) \psi^{*}(a, b)^{x} d x
$$

Where $\psi^{*}$ is the complex conjugate of a given function $\psi$. This function can be chosen arbitrary as long as it obeys certain rules. The DWT (Discrete Wavelet Transform) is an implementation using a set of discrete scales and wavelet translations respecting certain rules and mathematical conditions, the dilation equation is an example.

$$
\phi(\mathrm{x})=\sum_{\mathrm{k}=-\infty}^{\infty} \mathrm{a}_{\mathrm{k}} \emptyset\left(\mathrm{S}_{\mathrm{x}}-\mathrm{k}\right)
$$

Where $S$ is a scale factor (usually a value of 2) and ak represents a finite set of coefficients defining the scale function as well as the wavelet itself.

A simple method to implement the DWT(Discrete Wavelet Transform) is to take the starting matrix, called $\mathrm{LL}_{0}$ (the image to be compressed) [9], and process as follows:

1) The coefficients are grouped into square of four.

2) The average of the four coefficients for each square is taken, the result is a matrix four times smaller than the $L L_{0}$, be called $L L_{1}$

3) Starting from the same squares, the difference between the upper coefficients and lower ones are set to average the two obtained results, a new matrix is be given called $\mathrm{LH}_{1}$. It is also four times smaller.

4) The difference is set once again, this time between the coefficients of the left side of the matrix and its right side. The result is stored in a sub-matrix called $\mathrm{HL}_{1}$.

5) Finally, a difference of the difference is established (instead of the average), be called the sub-matrix $\mathrm{HH}_{1}$ the original one.

Consequently, four matrices $\left(\mathrm{LL}_{1}, \mathrm{LH}_{1}, \mathrm{HL}_{1}, \mathrm{HH}_{1}\right)$ are obtained, four times smaller than

\section{HaarAlgorithm}

The Daubechies wavelets are the orthogonal wavelet basis used extensively [10]. There are 10, numbered 2 to 20 (for even integers). The D2 is the wavelet Haar.

An image in black and white can be seen as a set of pixels, each pixel represents a grey level. Modelling such image is possible by a square matrix of size equal to the image resolution. For a color image, it is enough to consider three images, each representing the level of red, green and blue of the original picture. 
There are an infinite number of multi-scale basis functions (wavelets), but for each type of a wavelet there is a function called "mother wavelet" [11].The wavelet family of sub-division is in this case the family of functions $\psi_{\mathrm{j}}^{\mathrm{i}}(\mathrm{x})$ for $0 \leq \mathrm{j} \leq 2^{\mathrm{i}}$ where $\psi(\mathrm{x})$ is the following function:

$$
\psi(x)=\left\{\begin{array}{l}
1 \text { if } 0 \leq x<\frac{1}{2} \\
-1 \text { if } \frac{1}{2} \leq x<1 \\
0 \text { otherwise }
\end{array}\right.
$$

Note that the Haar basis is constructed orthogonal and not orthonormal. To ensure the normality, we replace $\psi_{j}^{\mathrm{i}}(\mathrm{x})$ by $2^{\mathrm{i} \backslash 2} \psi_{j}^{\mathrm{i}}(\mathrm{x})$, because of the scalar product $\left(2^{\mathrm{i} \backslash 2} \Psi_{\mathrm{j}}^{\mathrm{i}}(\mathrm{x}), 2^{\mathrm{i} \backslash 2} \Psi_{\mathrm{j}}^{\mathrm{i}}(\mathrm{x})=1\right)$.

\section{Results\& Analysis}

The aim of our work is to perform the image data compression by using the Discrete Wavelet Transform (DWT) technique while keeping the essential quality of the original image, in other terms, having a good compromise between PSNR (Peak Signal to Noise Ratio) and the compression

The chosen wavelets for testing are classified by family. Every family has a set of wavelets whose number following the name indicates the order of the filters used for their designs.

The wavelets used in the test are :

1) Biorthogonale wavelets Bior2.2, Bior3.3, Bior4.4, Bior5.5 and Bior6.8.

2) Daubechies wavelets: Haar, Db2, Db3, Db4, Db5, Db6, Db7, Db8, Db9, Db10 and Db11.

3) Coiflets wavelets: Coif1 and Coif4.

These wavelets are considered compactly supported which corresponds a Finite Impulse Response (FIR).

Even though, the quality of image compression systems is influenced by the content of the image or the spectral activity. The choice of an image for compression tests is a fundamental problem $[12,13]$, that's why images must be classified according to their frequency and spatial characteristics.

Spatial Frequency Measure (SFM) is a measure that gives a general idea of spectral activity of the image [14].
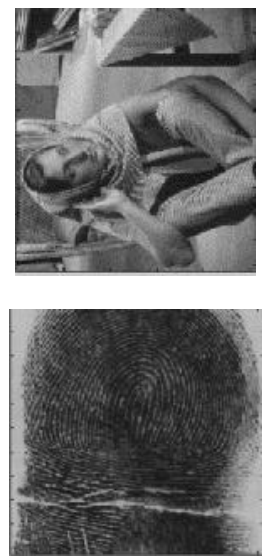

(a)
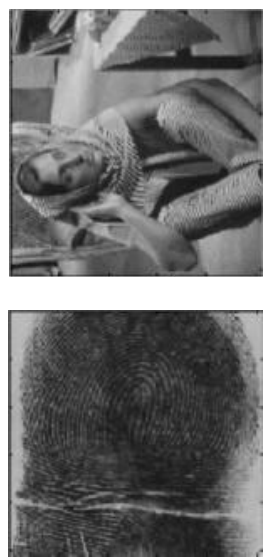

(b)
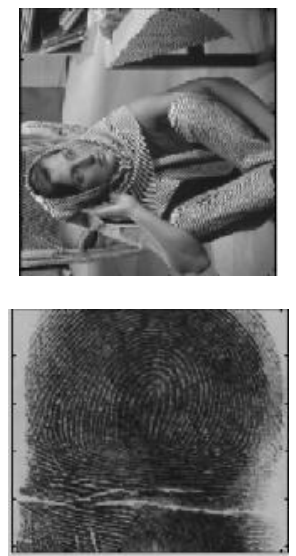

(c)
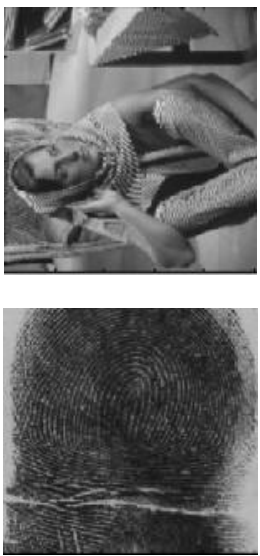

(d)
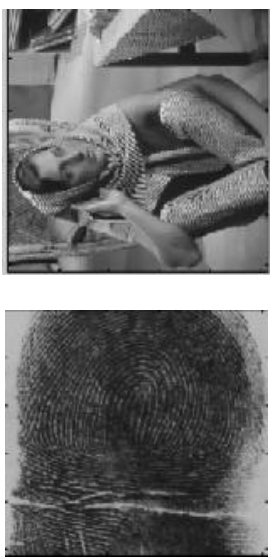

(e)

Figure 1. Image compression 'Woman' by using the wavelets of:

"dct","haar","coiflet","Daubechies". (a): originale image ; (b): Compression by DCT; (c): Compression DWT(discrete wavelet transform) by "Daubechies"; (d) Compression dwt by "coiflet"; (e): Compression DWT by "haar" 
Figure 1 illustrates the results given by DWT (discrete wavelet transform) of Daubechies in comparison to other wavelets. The DWT (discrete wavelet transform) of Daubechies gives good results.

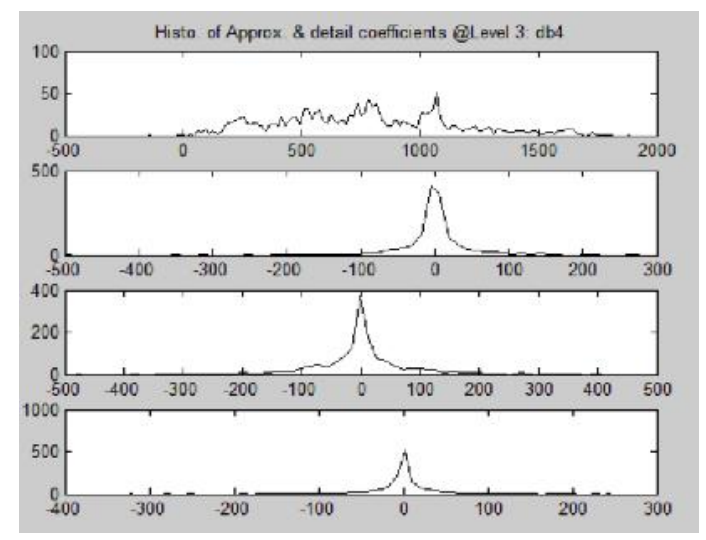

Figure 2. Histograms of the DWT(discrete wavelet transform) coefficents of the $3 d$ level of the Lennaimage by wavelets of

Daubechies2 "db2". (a) histogram of the coefficients of approximation $\mathrm{LL}_{3}$, (b)

histogram of detail coefficients $\mathrm{HL}_{3}$, (c) histogram $\mathrm{LH}_{3}$ detail coefficients, (d) histogram $\mathrm{HH}_{3}$ detail coefficient

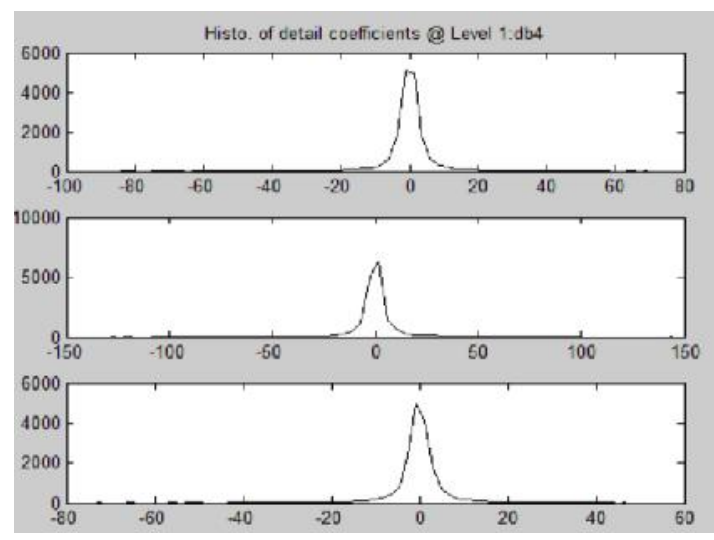

Figure 3. Histograms of DWT coefficients of the first level of the Lena image by wavelet

Daubechies2 "db2". (a) histogram of the coefficients of approximation $\mathrm{LL}_{3}$, (b) histogram of detail coefficients $\mathrm{HL}_{3}$, (c) histogram of detail coefficients $\mathrm{LH}_{3}$, (d) histogram of detail coefficients $\mathrm{HH}_{3}$

From a compression point of view, energy compacted within DWT coefficients (discrete wavelet transform) allows to obtain a high degree of image compression. According to the above figures, we calculated the amplitudes of DWT(discrete wavelet transform) coefficients at different scales and orientations. We note that all the details of coefficients histograms that resemble the double exponential distribution (Figure 2, 3).

Table1. Percentage of the total energy in the DWT coefficients for the Lenna image

\begin{tabular}{llllll}
\hline Level & Db15 & Haar & Coif2 & Db4 & Bior2.2 \\
\hline $\mathbf{L L}_{3}$ & 98.12 & 95.25 & 97.23 & 97.30 & 96.13 \\
$\mathbf{H L}_{3}$ & 0.0295 & 0.2319 & 0.0736 & 0.0819 & 0.0865 \\
$\mathbf{L H}_{3}$ & 0.0848 & 0.5579 & 0.1902 & 0.2250 & 0.1953 \\
$\mathbf{H H}_{3}$ & 0.0213 & 0.1008 & 0.0455 & 0.0524 & 0.0257 \\
$\mathbf{H L}_{2}$ & 0.0660 & 0.3606 & 0.1458 & 0.1544 & 0.2592 \\
$\mathbf{L H}_{2}$ & 0.2184 & 0.9346 & 0.4576 & 0.4961 & 0.6851 \\
$\mathbf{H H}_{2}$ & 0.0613 & 0.1735 & 0.1100 & 0.1268 & 0.1379 \\
$\mathbf{H L}_{1}$ & 0.2303 & 0.4770 & 0.4820 & 0.3482 & 0.5386 \\
$\mathbf{L H}_{1}$ & 1.0090 & 1.6111 & 1.0795 & 0.9877 & 1.6149 \\
$\mathbf{H H}_{1}$ & 0.1591 & 0.3050 & 0.1842 & 0.2271 & 0.3289 \\
\hline
\end{tabular}

"Table 1" shows the percentage of energy to 3 levels, each level and each orthogonal orientation using Haar wavelets 'Haar';Daubechies "db4", "db15" and Coiflet "coif2" and wavelets bi- orthogonal "bior2.2".

It is noted that Daubechies wavelet compacts the energy in the approximation coefficients than other wavelets.

It can be inferred that the orthogonal wavelets establishes a better and a more satisfying percentage than the bi-orthogonal wavelets in terms of energy compaction. We also note that almost all the energy is in the approximation coefficients which mean that a high degree of compression has been achieved. 
Conserving energy is an important property of an orthogonal transformation. This also implies that the total distortion due to quantization of DWT(discrete wavelet transform) coefficients is equal to the sum of the distortions in each sub-band. Therefore, it allows quantifying the individual sub-bands at different levels in order to achieve a high level of compression and a good visual quality.

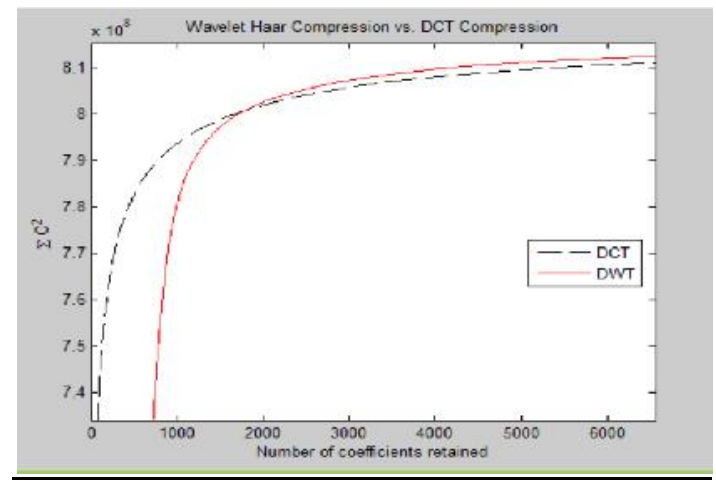

Figure 4. Comparison between the DWT compression of Haar and DCT (discrete cosine transform) to the energy leve

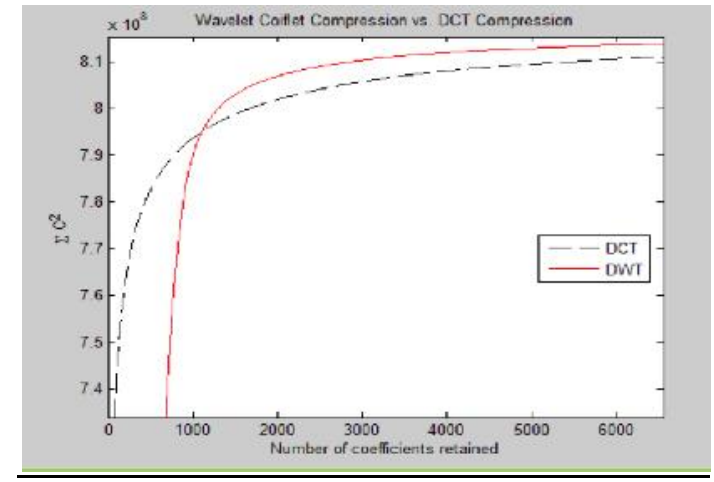

Figure 5. Comparison between the DWT compression of Coiflet and DCT to the energy level

Figure 4 and 5 prove that the compacted energy is proportional to the number of coefficients, when increasing the number of coefficients, the power also increases. It may be noted that the DWT outperforms DCT in terms of energy and reaches a maximum value of $8.1 \times 10^{8} \mathrm{~dB}$.

If the wavelet 'haar'and the 'coiflet' compared, we can say that they have a likely behaviour but with a slight difference.

For the evaluation of the image quality after compression, two coefficients are calculated which are: PSNR(Peak signal to noise ratio) and compression rate (CR).

PSNR (Peak Signal Noise Ratio) is a simple and effective measure distortion [4].

$$
\begin{aligned}
& \text { MSE }=\frac{1}{p} \sum_{i=0}^{p-1}\left(a_{i}-b_{i}\right)^{2}(4) \\
& \text { PSNR }=10 \log _{10}\left(\frac{255^{2}}{M S E}\right)
\end{aligned}
$$

Table 2 illustrates the results of compression of two images by the wavelet transform. The wavelets used for image compression wavelets are the bi-orthogonal wavelets.

Table 2. PSNR (Peak Signal Noise Ratio) and TR (compression rate) compressed images by biorthogonal wavelets

\begin{tabular}{ccccccc}
\hline wavelet & \multicolumn{3}{c}{ woman } & \multicolumn{3}{c}{ fingerprint } \\
& PSNR & MSE & TC & PSNR & MSE & TC \\
\hline Bior2.2 & 29.5966 & 71.91 & 73.9 & 27.3042 & 121.91 & 87.8 \\
Bior3.3 & 29.188 & 78.99 & 71.9 & 26.6496 & 141.75 & 85 \\
Bior3.7 & 29.7504 & 69.41 & 71 & 27.1316 & 126.86 & 83.9 \\
Bior4.4 & 31.9355 & 52.84 & 74.1 & 28.7986 & 172.43 & 88.5 \\
Bior5.5 & 31.0456 & 51.51 & 73 & 25.7967 & 172.51 & 88.8 \\
Bior6.8 & 31.1229 & 50.60 & 73.6 & 26.0916 & 161.18 & 87.3 \\
\hline
\end{tabular}

For different levels of bi-orthogonal wavelets, different PSNR (Peak Signal Noise Ratio) exist. Regarding table, the compression of the image "woman" by different bi-orthogonal waveletsshows a variation of the PSNR (Peak Signal Noise Ratio) from 29.188 dB to 31.9355 $\mathrm{dB}$. While, on the other hand, the compression of the fingerprint image with the same variation of the wavelet provides a PSNR from $25.7967 \mathrm{~dB}$ to $28.7986 \mathrm{~dB}$. The fingerprint image has a 
PSNR (Peak Signal Noise Ratio) very low compared to the PSNR (Peak Signal Noise Ratio) of the image "woman". This is due to the fact that the fingerprint image has more spectral activity compared to the image "woman". It can be inferred that the higher spectral activity is, the lower PSNR (Peak Signal Noise Ratio).

"Table 3" presents the evaluation of PSNR (Peak Signal Noise Ratio) [15] and the CT image compression based on different levels of Daubechies wavelet.

PSNR (Peak Signal Noise Ratio) of the image compression "woman" by various Daubechies wavelets varies between $30.7 .97 \mathrm{~dB}$ and $31.7695 \mathrm{~dB}$. The change in PSNR for different wavelet used is almost uniform. This can be explained by the fact that the image "woman" does not have a lot of variations of spectral activity.

As the spectral activity of the fingerprint image is more important than the image "woman", so it makes sense for different Daubechies wavelets, the values of PSNR (Peak Signal Noise Ratio) of the image are lower "finguer" to those of the image woman. PSNR (Peak Signal Noise Ratio) of the fingerprint image varies from $26.7570 \mathrm{~dB}$ to $30.6616 \mathrm{~dB}$.

Table 3. PSNR (Peak Signal Noise Ratio) and TR (compression rate) compressed images by Daubechies wavelets

\begin{tabular}{cccccccc}
\hline wavelet & \multicolumn{3}{c}{ woman } & \multicolumn{3}{c}{ fingerprint } \\
& PSNR & MSE & TC\% & PSNR & MSE & TC \% \\
\hline Db2 & 31.7695 & 54.89 & 72 & 30.6616 & 56.28 & 86.9 \\
Db3 & 31.06 & 51.34 & 73.9 & 27.8302 & 108.01 & 87.73 \\
Db4 & 31.0703 & 51.22 & 73.6 & 26.7570 & 138.29 & 87.7 \\
Db5 & 31.0247 & 51.76 & 73.8 & 27.1581 & 126.09 & 86.9 \\
Db6 & 30.7097 & 55.66 & 73.4 & 26.8965 & 133.91 & 87.4 \\
Db7 & 30.9471 & 52.70 & 73 & 27.9275 & 105.61 & 87.1 \\
Db10 & 30.8685 & 53.66 & 71.9 & 28.7515 & 87.36 & 86.7 \\
\hline
\end{tabular}

The table above represent a comparison of image compression by biorthogonal wavelet and Daubechies. We can see that the best PSNR (Peak Signal Noise Ratio) is given by the wavelet Bior4.4 for woman image with a compression ratio of $74.1 \%$. This wavelet Bior4.4 also shows the best PSNR for the fingerprint image with a compression ratio of $88.5 \%$.

The second best PSNR (Peak Signal Noise Ratio) is given by the wavelet Bior6.8 with a compression ratio of $50.6 \%$ in the image of "woman" and $87.3 \%$ for the fingerprint image.

As the compression quality premium on the compression rate, except for some applications, we can say that with biorthogonales wavelets, it is better to use the Bior4.4 wavelet for the compression of a set of images[16]. Regarding "Table 3", it is clear that the wavelet Daubechies2 "Db2" gives the best PSNR (Peak Signal Noise Ratio) but with a compression rates ranking from $86.9 \%$ to $72 \%$. It is also noted from Table 3 that the wavelet Daubechies 3 "Db3" gives PSNR of $27.83 \mathrm{~dB}$ with a compression ratio of $87.73 \%$ for the fingerprint image, a PSNR $31.06 \mathrm{~dB}$ with a compression ratio of $73.9 \%$ for picture woman . In the family of Daubechies wavelets, wavelet "Db3" gives a very good compression rate.

"Figure 6" shows the influence of the decomposition level on the number of zeros and on the retaining power. Indeed, the results of the figure have a clear tendency, over the level of decomposition increases more than the percentage of zeros obtained also increases. Furthermore the decomposition at higher levels means that a higher proportion of sub-signals are input from the detail coefficients.

It is noticed that the retained energy was higher at higher levels of decomposition, it is because at higher levels of decomposition, there is a large proportion of the coefficients in the detail of sub-signals.

According to "Table 4", it can be deduced that the increased levels of decomposition is proportional with the increase in the percentage of zeros, but with a lower threshold. Therefore, this model of decomposition increases shows that more details will be lost in it. 


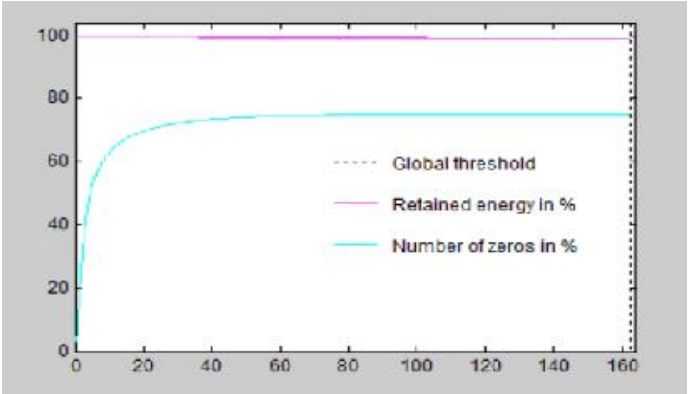

(a)

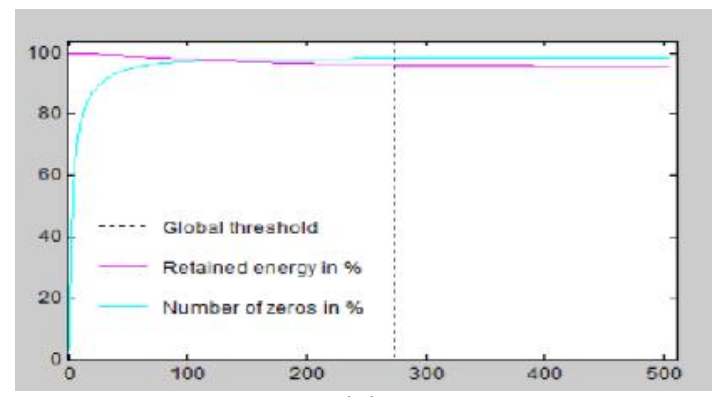

(c)

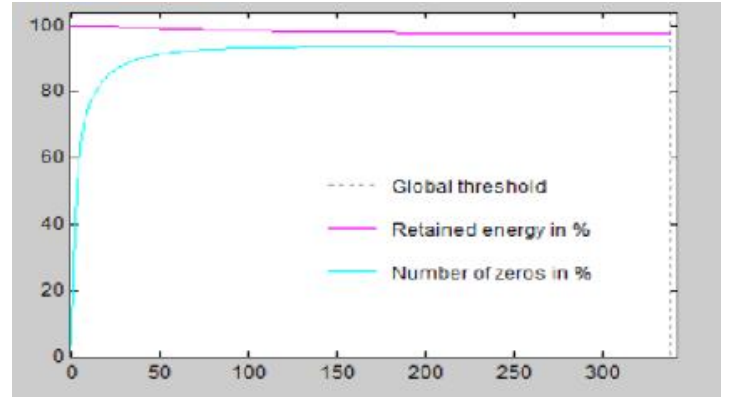

(b)

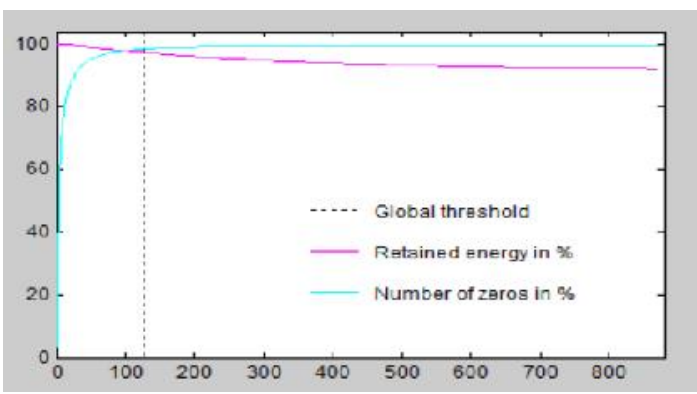

(d)

Figure 6. Percentage of energy retained and the number of zeros using different levels of decomposition for image 'woman' (a) first level of decomposition; (b) the second level of decomposition; (c) the third level of decomposition; (d) fourth level of decomposition

Table 4. Results for different levels of decomposition

\begin{tabular}{cccc}
\hline $\begin{array}{c}\text { Level of } \\
\text { decomposition }\end{array}$ & $\begin{array}{c}\text { Global } \\
\text { tresholding }\end{array}$ & $\begin{array}{c}\text { \% retained } \\
\text { energy }\end{array}$ & $\begin{array}{c}\% N^{\circ} \text { de } \\
\text { zeros }\end{array}$ \\
\cline { 1 - 3 } level 1 & 162.5 & 99.11 & 75 \\
level 2 & 338.8 & 97.64 & 93.75 \\
level 3 & 273.6 & 96.13 & 98.36 \\
level 4 & 128.5 & 97.42 & 98.56 \\
\hline
\end{tabular}

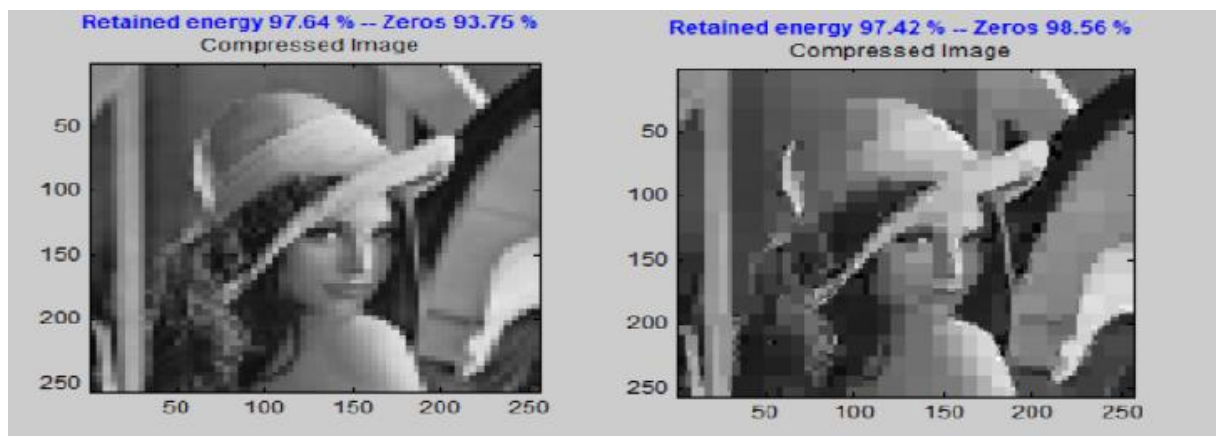

Figure 7. Compressed image of "lenna" to the second level and the fourth level

According to "Figure 7", we note that the compressed image quality decreases as a function of level increase meaning a very high number of zeros.

To improve the quality of compression DWT (discrete wavelet transform), we showed significant improvements especially when the number of coefficients used for reconstruction of the image is very low. Yet the higher decomposition levels are responsible for greater compression, but it affects the retained energy in the synthesized image.Results also cover the affects of decomposition level on the proportion of detail coefficients to an extent. It is also 
noticed that the details was extra at higher level of decomposition that can be thresholded to gain higher compression rates.Nevertheless acquiring higher compression rates will conduct to energy loss, therefore to achieve of best compromise between compression ratio and energy compaction is at lower decomposition level (two or three level decomposition).

\section{Conclusion}

Image compression is the subject of many studies that focus on improving compression algorithms as well as the development of new techniques and compression formats. In this paper, we studied the JPEG compression standards (Joint Photographic Experts Group) andspecifically JPEG2000 (Joint Photographic Experts Group2000) or wavelet compression. The use of wavelet allows many applications at the image compression. Several wavelet families are currently used, and offer better compression result, where values can be neglected below a certain threshold. This does not alter the recognition of the components of the image.The image display can be gradually done. This may be necessary for transmission media at low flow. The choice of wavelet depends on the application. In some cases, the simplest wavelet (Haar), which has a quick and easy calculation will be the most optimal. For other applications, this is the worst possible choice.

\section{References}

[1] Frédéric Dufaux. Compression d'images par ondelettes. TELECOM ParisTech. 2011.

[2] DS Taubman, MW Marcellin. JPEG2000: Image compression fundamentals, standardsand practice. Kluwer Academic Publishers. Boston. 2002.

[3] M Rabbani, R Joshi. An overview of the JPEG 2000 still image compression standard. Elsevier, Signal processing: Image communication. 2002: 3-48.

[4] YQ SHi, H Sun. Image and video compression for multimedia engineering. 2nd Ed. CRC press, Taylor \& Francis group. 2008.

[5] V Chandrasekhar, G Takacs, D Chen, SS Tsai, J Singh, B Girod. Transform coding of image feature descriptors. Proc. SPIE, Visual Communications and Image Processing. 2009; 7257.

[6] Yun Q Shi, Huifang Sun. Image and video compression for multimedia engineering. Fundamentals, Algorithms, and Standards. 2nd ed. 2008.

[7] Avanaki M, Ahmadinejad H, Ebrahimpour R. Evaluation of Pure Fractal and Wavelet Fractal Compression Techniques. ICGST-GVIP Journal. 2009; 9(4): 41-47.

[8] Marco Cagnazzo. JPEG et JPEG2000: étude de cas. TELECOM ParisTech. 2010.

[9] Ruchika, Mooninder Singh, Anant Raj Singh. Compression of Medical Images Using Wavelet Transforms. International Journal of Soft Computing and Engineering (IJSCE). 2012; 2(2).

[10] I Daubechies. Orthonormal bases of compactly supported wavelets. Communication on Pure and Applied Mathematics. 1988; 41(7): 909-996.

[11] Kaleka Jashanbir Singh, Sharma Reecha. Comparativ performance analysis of Haar,Symlets and Bior wavelets on image compression using Discrete wavelet Transform. International journal of Computers and Distrbuted Systems. 2012; 1(2).

[12] GK Kharate, VH Patill, NL Bhale. Selection of mother wavelet for image compression on basis of nature of image. Journal of multimedia. 2007; 2(6).

[13] MK Mandal, S Panchanathan, T Aboulnasr. Choice of wavelets for image compression. Lecture note in computer science. 1996; 1133: 239-249.

[14] R Sakuldee, S Udomhunsakul. Objective Performance of Compressedlmage Quality Assessments. World Academy of Science, Engineering and Technology. 2007; 1(11): 11-29.

[15] Gérard Blanchet, Maurice Charbit. Digital Signal and Image Processing using MATLAB. ISTE Ltd. 2006.

[16] M Beladgham, A Bessaid, A Moulay-Lakhdar, M Ben Aissa, A Bassou. MRI Image Compression using Biorthogonal CDF Wavelet Based on Lifting Scheme and SPIHT coding. J. Sci. Res. 2010; 2: 225232. 